\title{
Supergravity Has Positive Energy
}

\author{
S. Deser \\ Department of Physics, Brandeis University, Waltham, Massachuestts 02154 \\ and \\ Claudio Teitelboim \\ Joseph Henry Laboratories, Princeton University, Princeton, New Jersey 08540
}

(Received 9 June 1977)

\begin{abstract}
We show that the total energy in supergravity theory is nonnegative. The derivation is based on the fact that for bounded systems the Hamiltonian operator is the square of the spinor supercharge just as for globally supersymmetric systems. The result holds for any of the pure, matter-coupled, or extended versions of supergravity, except those with cosmological term for which the concept of total energy does not exist.
\end{abstract}

One of the major unsolved problems in general relativity is the question of positivity of the total energy. Although many suggestive partial results have been given, ${ }^{1}$ the nonlinearity of the theory has frustrated complete demonstration of this important physical requirement. We show here that extension of general relativity to the more complex system of supergravity ${ }^{2}$ leads to a compact demonstration of the positivity property for this wider system, at the second-quantized level.

We first recall the definition and essential properties of the energy for a generally covariant system. Energy is defined for (and only for) asymptotically flat spaces where Poincaré group is a symmetry at spacelike infinity. The numerical value of the total energy (including that of matter) is given by an integral over a two-dimensional surface at spatial infinity ${ }^{3}$ of a combination of derivatives of the spatial metric components; in asymptotically Cartesian coordinates, it reads

$$
P^{0}=(16 \pi G)^{-1} \oint_{r \rightarrow \infty} d^{2} s_{i}\left(g_{i j, j}-g_{j j, i}\right),
$$

and similar expressions hold ${ }^{3,4}$ for the total momentum and angular momentum. The metric components in (1) are not independent but are constrained by the initial-value equations $G_{0 \mu}-T_{0 \mu}$ $=0$. These flux-integral expressions are in close parallel with the familiar ones for charges in vector gauge theories.

Although one may evaluate the energy of any particular field configuration by means of (1), it is not possible to use expression (1) naively as the generator of time translations. It is first necessary to fix the gauge (i.e., to pick a preferred local time) by means of coordinate conditions in order to be able to take meaningful commutators of $P^{0}$ with the various fields of the the- ory. 3,4 The reason for this necessity may be understood as follows: After coordinate conditions are imposed (and only then) the surface on which the state is defined is totally determined once its asymptotic location is given. Therefore if the surface is deformed, the magnitude of the deformation at infinity determines the deformation everywhere. That is, once the local gauge is fixed, there remains only the global gauge freedom associated with the specification of the gauge functions at infinity. If the deformation is asymptotically a timelike translation of the surface parallel to itself, the generator of the corresponding global deformation is precisely $P^{0}$ which is called then the reduced Hamiltonian (because the theory has been "reduced" by the coordinate conditions). Similar remarks hold for the other generators of the Poincaré group.

If the gauge is fixed, the naive commutation rules of the dynamical variables change according to the general method of Dirac. ${ }^{5}$ The new commutators are the quantum analogs of the Dirac bracket and in terms of them the surface integrals giving $P_{\mu}$ and $J_{\mu \nu}$ satisfy (as they should) the algebra of the generators of the Poincaré group $^{4}$ irrespective of the choice of local gauge. In simple cases $^{3,4}$ the gauge is fixed by setting some of the canonical coordinates equal to zero and by expressing their conjugate momenta as functionals of the remaining independent degrees of freedom by means of the constraint equations $G_{0 \mu}-T_{0 \mu}=0$. In that case the new commutators are simply the old ones in terms of the independent canonical variables. Although formal arguments exist $\mathrm{t}^{3,6}$ to show that one can indeed solve for the redundant momenta, the actual solution cannot be exhibited in closed form because of the 
highly nonlinear nature of the equations. Therefore it is hard to obtain information on the positivity of the reduced Hamiltonian in this direct way. In supergravity the complexity of the equations is even greater and one still cannot solve the constraints in closed form. However, on account of the supersymmetry invariance of the theory we can show algebraically that the reduced Hamiltonian is nonnegative.

The surface integrals giving $P_{\mu}$ and $J_{\mu \nu}$ are the same functions of the gravitational variables alone for supergravity as for ordinary general relativity. However, in the former a new surface integral arises on account of the invariance of the theory under local supersymmetry transformations: the total supercharge, ${ }^{7,8}$

$$
S=\frac{1}{2} \oint d^{2} s_{i}\left[\gamma^{i}, \gamma^{j}\right] \dot{\psi}_{j},
$$

which is a Majorana spinor. ${ }^{9}$ Again here $S$ cannot be used as a generator unless the supergauge is fixed (for example by the natural condition $\gamma^{i} \psi_{i}=0$ ). After the gauges are fixed the commutators (or anticommutators in the case of two Fermi variables) of the various fields in the theory are no longer the naive ones but correspond rather to the (graded) Dirac bracket. In the new commutators the surface integrals $S_{A}, P_{\mu}$, and $J_{\mu \nu}$ can be meaningfully used as generators ${ }^{7}$ and satisfy the usual Minkowski-space supersymmetry algebra, ${ }^{10}$ as can be shown from the closure relations of the constraints of the theory. ${ }^{11}$ In particular one has

$$
S_{A} S_{B}+S_{B} S_{A}=16 \pi G \hbar\left(\gamma^{\mu}\right)_{A B} P_{\mu}
$$

Multiplying (3) by $\gamma_{0}$ and taking the trace we immediately obtain

$$
P^{0}=(8 \pi G \hbar)^{-1} \sum_{A=1}^{4} S_{A}^{2},
$$

which shows that the total-energy operator of supergravity is nonnegative. It is important in connection with this statement to recall that (i) the $S_{A}$, being Majorana spinors, are Hermitian, and (ii) since the gauge has been fixed there are no ghosts in the Hilbert space and therefore the inner product is positive definite.

The above simple proof holds as well for the two important generalizations of pure supergravity. The first is the inclusion of locally supersymmetric couplings to matter. There, as in ordinary gravity, the transformation properties of the surface integrals under asymptotic Poincaré and supersymmetry transformations are not changed by the presence of (bounded) matter sources. The rapid falloff of the sources is not an additional assumption, being already necessary to ensure that the surface integrals exist. ${ }^{12}$ As a consequence, relation (3) is still valid, and the proof applies as it stands. The second and perhaps most important is extended supergravity. There, in addition to the obvious replacement of $S_{A}$ by $S_{A i}$ and of $\left(\gamma^{\mu}\right)_{A B}$ by $\left(\gamma^{\mu}\right)_{A B} \delta_{i j}$, extra terms do arise in general on the right-hand side of (3), corresponding to internal central gauge charges. Hence no $\gamma$ matrices appear in those terms as no space-time index is available to contract with. Therefore upon multiplication by $\gamma_{0}$ and taking the trace the extra pieces drop out. Equation (4a) is then modified to read

$$
P^{0}=(8 \pi G \hbar N)^{-1} \sum_{i=1}^{N} \sum_{A=1}^{4} S_{A i}{ }^{2},
$$

where $i$ labels the internal generators. Therefore $P^{0}$ is still nonnegative in extended supergravity.

It should be emphasized that the above positivity argument is meaningful only within the context of quantum field theory where, in particular, the vector-spinor $\psi_{i}$ is regarded as a quantum field satisfying the equal-time anticommutation rules $^{8,13}$

$$
\begin{aligned}
\psi_{i A}(x) \psi_{j B}\left(x^{\prime}\right) & +\psi_{j B}\left(x^{\prime}\right) \psi_{i A}(x) \\
& =\hbar g^{-1 / 2}\left(\gamma_{j} \gamma_{i}\right)_{A B} \delta\left(x, x^{\prime}\right),
\end{aligned}
$$

rather than an "anticommuting $c$-number." In the classical limit the $\psi_{i}$ field disappears and supergravity goes over to ordinary general relativity. However, in that same limit both sides of (3) vanish for any $P_{\mu}$ and nothing can be concluded about the energy. Put in more physical terms, our proof of postivity of the energy requires inclusion of the fluctuations of the spin- $\frac{3}{2}$ field, a purely quantum-mechanical phenomenon which is not present in the classical limit. ${ }^{14}$ It is also appropriate to remark here that quantized ordinary general relativity is not a special case of supergravity as there is no coupling constant which could be set equal to zero in order to decouple the $\psi_{i}$ field (the coupling is of gravitational strength). Hence the positivity proof does not say anything either about the energy operator of pure quantum gravity. This is of course just another way of stating that the key elements are the fluctuations of $\psi$ which are neglected in ordinary general relativity.

The proof presented in this note here takes for 
granted (as is done for all interacting systems!) that a Hilbert space can be constructed such that the operators $P^{0}$ and $S_{A}$ exist and are Hermitian. ${ }^{15}$ This is a most difficult problem indeed, but we believe that, even at this heuristic level, our arguments provide additional evidence for the attractiveness of supergravity as an extension of general relativity.

This work is supported by National Science Foundation Grants No. PHY-76-07299 to Brandeis University and No. PHY-76-82662 to Princeton University. One of us (C.T.) acknowledges a research fellowship from the Alfred P. Sloan Foundation.

${ }^{1}$ See, for example, D. Brill and S. Deser, Ann. Phys. (N.Y.) 50,548 (1968); D. Brill, S. Deser, and L. D. Fadeev,.Phys. Lett. 26A, 538 (1968); N. Ó Murchadha and J. W. York, Phys.Rev. D 10, 2345 (1974); P.S. Jang, J. Math. Phys. (N.Y.) 17, 141 (1976); Y. Choquet and J. Marsden, C. R. Acad. Sci. 282, 609 (1976).

${ }^{2}$ D. Z. Freedman, P. van Nieuwenhuizen, and S. Ferrara, Phys. Rev. D 13, 3214 (1976); S. Deser and B. Zumino, Phys. Lett. 62B, 335 (1976).

${ }^{3}$ R. Arnowitt, S. Deser, and C. W. Misner, Phys. Rev. $117,1595(1960)$, and 118, $1100(1960)$, and 122,997 (1961), and in Gravitation: An Introduction to Current Research, edited by L. Witten (Wiley, New York, 1962).

${ }^{4}$ T. Regge and C. Teitelboim, Phys. Lett. 53B, 101 (1974), and Ann. Phys. (N.Y.) 88, 286 (1974).

${ }^{5}$ P. A. M. Dirac, Can. J. Math. 2, 129 (1950). See also A. Hanson, T. Regge, and C. Teitelboim, Constrained Hamiltonian Systems (Accademia Nazionale dei Lincei, Roma, 1976).

${ }^{6}$ C. Teitelboim, Phys. Lett. 56B, 376 (1975); M. Pilati and C. Teitelboim, in Proceedings of the Marcel Grossman Meeting, edited by R. Ruffini (North-Holland, Amsterdam, 1977).

${ }^{7} \mathrm{C}$. Teitelboim, to be published.

${ }^{8}$ S. Deser, J. H. Kay, and K. S. Stelle, Phys. Rev. D (to be published).

${ }^{9}$ One may require $\psi_{i} \sim r^{-2}$ for large distances in order to ensure that $S$ be finite. This asymptotic behavior is compatible (in the sense that the constraints are asymptotically satisfied) with the usual boundary conditions (Refs. 3 and 4) on the gravitational variables which make $P_{\mu}$ and $J_{\mu \nu}$ finite.

${ }^{10} \mathrm{~J}$. Wess and B. Zumino, Nucl. Phys. B70, 39 (1974), and Phys. Lett. 49B, 52 (1974).

${ }^{11}$ C. Teitelboim, Phys. Rev。 Lett. 38, 1106 (1977). It should be emphasized that the total charges are not the integrals of the corresponding local constraints, since the latter vanish. However, it may be shown that the (nonzero) total charges do close in the same manner as the (vanishing!) integrated constraints do. This is accomplished by expressing the asymptotic part of the commutator of two transformations as a function of the asymptotic part of the original transformations.

${ }^{12}$ There is one interesting case, nevertheless, in which the matter contribution is crucial. Consider the cosmological extension of supergravity [D. Z. Freedman and A. Das, to be published; S. MacDowell and F. Mansouri, Phys. Rev. Lett. 38, 739 (1977); P. K. Townsend, State University of New York at Stony Brook Report No. ITP-SB-77-2 (to be published)]. The presence of a cosmological term excludes asymptotically flat boundary conditions and the very concept of energy. However, it has recently been observed [S. Deser and B. Zumino, Phys. Rev. Lett. 38, 1433 (1977)] that this cosmological term, when used to compensate one of opposite sign arising from spontaneous symmetry breaking, led to a theory without net cosmological term, but with a Goldstone fermion. Although we have not yet analyzed this situation, it seems quite possible in view of the resulting asymptotic flatness that our present consideration will apply in that case as well when due care is taken with the cosmological behavior of the Goldstone fermion action. Should this be the case, energy positivity will persist also in the special gauge in which the Goldstone field is removed and the spin- $\frac{3}{2}$ field acquires a mass, simply because the energy commutes with the supercharge and so is supersymmetry invariant.

${ }^{13} \mathrm{R}$. Tabensky and C. Teitelboim, to be published; M. Pilati, to be published. The anticommutation rule (5) is related to the fact that supergravity is the square root of general relativity (Ref. 11), much in the same way as $\left\{\gamma_{\mu}, \gamma_{\nu}\right\}=2 \eta_{\mu \nu}$ is related to the fact that the Dirac equation is the square root of the Klein-Gordon equation. It should be pointed out that (5) holds before all gauges are fixed and it is changed (according to Dirac's procedure) after gauge conditions are imposed.

${ }^{14}$ That positive energy of interacting globally supersymmetric systems is guaranteed by (3) has long been understood; B. Zumino, in Proceedings of the Seventeenth Intermational Conference on High Energy Physics, London, England, 1974, edited by J. R. Smith (Rutherford High Energy Laboratory, Didcot, Berkshire, England, 1975).

${ }^{15}$ Quantization might introduce anomalies in the algebra of local generators (Ref. 11), such as the ones responsible for the critical dimension in the quantum string. On the other hand, the present case differs from the string in that the algebraic properties of the total charges are determined by the asymptotic aspects of a local gauge group, which might preserve (4) even if anomalies appear in the local algebra. A related issue is whether a cosmological term will be induced by closed loops, and so destroy asymptotic flatness. A favorable indication is given by explicit calculations in globally supersymmetric systems [B. Zumino, Nucl. Phys. B89, 535 (1975)] where the pure vacuum diagrams cancel, even using "old-fashioned" regularization, so that no cosmological term appears. But formally at least, supergravity is globally supersymmetric after 
the gauge is fixed up to boundary conditions, and the above results are encouraging. One may also argue that direct closed loop calculations will not produce a cosmological term either, simply because dimensional regularization (which respects the gauge invariances) leads to vanishing of tadpole diagrams.

\title{
Observation of a Dimuon Resonance at 9.5 GeV in 400-GeV Proton-Nucleus Collisions
}

S. W. Herb, D. C. Hom, L. M. Lederman, J. C. Sens, ${ }^{(a)}$ H. D. Snyder, and J. K. Yoh

Columbia University, New York, New York 10027

and

J. A. Appel, B. C. Brown, C. N. Brown, W. R. Innes, K. Ueno, and T. Yamanouchi

Fermi National Accelerator Laboratory, Batavia, Illinois 60510

and

A. S. Ito, H. Jöstlein, D. M. Kaplan, and R. D. Kephart

State University of New York at Stony Brook, Stony Brook, New York 11974

(Received 1 July 1977)

Accepted without review at the request of Edwin L. Goldwasser under policy announced 26 April 1976

\begin{abstract}
Dimuon production is studied in $400-\mathrm{GeV}$ proton-nucleus collisions. A strong enhancement is observed at $9.5 \mathrm{GeV}$ mass in a sample of 9000 dimuon events with a mass $m_{\mu^{+} \mu^{-}}$ $>5 \mathrm{GeV}$.
\end{abstract}

We have observed a strong enhancement at 9.5 $\mathrm{GeV}$ in the mass spectrum of dimuons produced in $400-\mathrm{GeV}$ proton-nucleus collisions. Our conclusions are based upon an analysis of $9000 \mathrm{di}-$ muon events with a reconstructed mass $m_{\mu^{+} \mu^{-}}$ greater than $5 \mathrm{GeV}$ corresponding to $1.6 \times 10^{16}$ protons incident on $\mathrm{Cu}$ and $\mathrm{Pt}$ targets:

$$
p+(\mathrm{Cu}, \mathrm{Pt}) \rightarrow \mu^{+}+\mu^{-}+\text {anything. }
$$

The produced muons are analyzed in a doublearm magnetic-spectrometer system with a mass resolution $\Delta m / m(\mathrm{rms}) \approx 2 \%$.

The experimental configuration (Fig. 1) is a modification of an earlier dilepton experiment in the Fermilab Proton Center Laboratory. ${ }^{1-3} \mathrm{Nar}-$ row targets $(\sim 0.7 \mathrm{~mm})$ with lengths corresponding to $30 \%$ of an interaction length are employed.

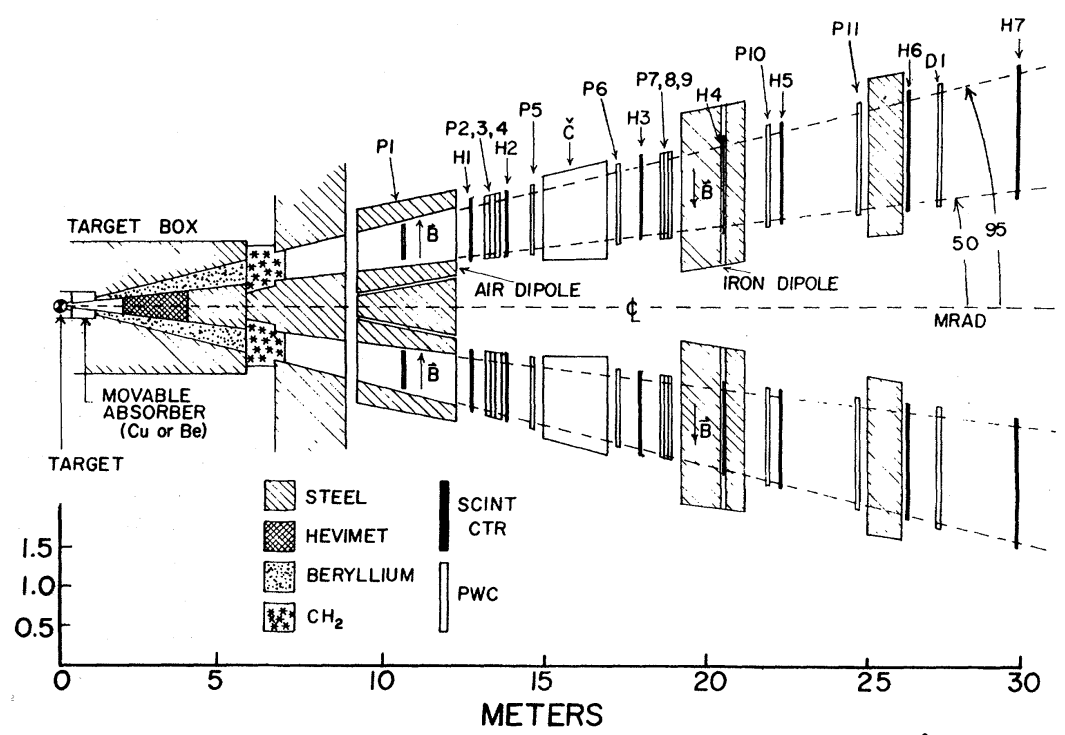

FIG. 1. Plan view of the apparatus. Each spectrometer arm includes eleven PWC's P1-P11, seven scintillation counter hodoscopes $\mathrm{H} 1-\mathrm{H} 7$, a drift chamber D1 and a gas-filled threshold Čerenkov counter Č. Each arm is up/ down symmetric and hence accepts both positive and negative muons. 